\title{
Unusual Properties of Superoscillating Particles
}

\author{
Achim Kempf \\ Department of Applied Mathematics, University of Waterloo \\ Waterloo, Ontario N2L 3G1, Canada \\ Paulo J. S. G. Ferreira \\ Dept. Electrónica e Telecomunicacões / IEETA \\ Universidade de Aveiro, 3810-193 Aveiro, Portugal
}

\begin{abstract}
It has been found that functions can oscillate locally much faster than their Fourier transform would suggest is possible - a phenomenon called superoscillation. Here, we consider the case of superoscillating wave functions in quantum mechanics. We find that they possess rather unusual properties which raise measurement theoretic, thermodynamic and information theoretic issues. We explicitly determine the wave functions with the most pronounced superoscillations, together with their scaling behavior. We also address the question how superoscillating wave functions could be produced.

PACS numbers: 03.65.Ta,03.67.-a,02.30.Nw
\end{abstract}

\section{INTRODUCTION}

Consider a differentiable function which contains, according to its Fourier transform, only wavelengths larger than some finite minimum wavelength $\lambda_{\min }$. Intuitively, one expects that this function cannot oscillate on length scales significantly smaller than $\lambda_{\min }$. In fact, however, it has been found that such functions are able to oscillate locally with wavelengths that are arbitrarily smaller than $\lambda_{\text {min }}$. The phenomenon is known as superoscillation, and indications of its occurrence have been discussed in various contexts, from evanescent waves and seeming superluminal propagation to the transplanckian problem of black holes, see [1]- 7]. In the field of information theory, see e.g. [8, 9], the phenomenon of superoscillating functions had been observed even earlier, see [10].

In this Letter, we investigate the rather unusual properties of quantum mechanical wave functions which superoscillate. For example, superoscillating particles tend to absorb energy from objects, thereby acting as "cooling-rays". Superoscillations come at a price, however, which is that they are generally of small amplitude. Thus, our aim here is to calculate and investigate the wave functions which possess the most pronounced superoscillations, building on the methods of $7,10,11$.

We find that the maximum amplitude of superoscillations decreases polynomially with the frequency and exponentially with the duration of the superoscillating stretch. We find that this scaling behavior is consistent with information theoretic considerations. Finally, we propose a method by which, in principle, superoscillating wave functions might be produced experimentally.

To be precise, let us consider the set of wave functions with momentum cutoff $p_{\max }$ :

$$
\psi(x)=\frac{1}{\sqrt{2 \pi \hbar}} \int_{-p_{\max }}^{p_{\max }} \tilde{\psi}(p) e^{\frac{i x p}{\hbar}} d p
$$

Such wave functions are linear combinations of plane waves whose wavelengths are at least as large as $\lambda_{\min }=$ $h / p_{\max }$. Nevertheless, as was shown in [7], such wave functions can oscillate arbitrarily quickly on arbitrarily long stretches, in the following sense: For any $N$ arbitrarily chosen points $\left\{x_{k}\right\}_{k=1}^{N}$ and arbitrarily chosen amplitudes $\left\{a_{k}\right\}_{k=1}^{N}$, there exist square integrable wave functions $\psi$ which at the prescribed points take the prescribed amplitudes

$$
\psi\left(x_{k}\right)=a_{k}, \quad \text { for all } \quad k=1,2, \ldots, N
$$

while also obeying the momentum cutoff expressed in Eq11 Note that $\psi$ will be differentiable, as is every wave function $\psi$ of the form given in Eq1] Note also that we are not yet normalizing $\psi$.

If we choose the spacing of the points $\left\{x_{k}\right\}$ to be small compared to the minimum wavelength, $x_{k+1}-$ $x_{k} \ll \lambda_{\min }$, while choosing oscillatory amplitudes $\left\{a_{k}\right\}$, e.g. $a_{k}=(-1)^{k}$, then we say that the resulting wave function $\psi$ is superoscillating. Intuitively, the superoscillations do not show as high frequencies in the Fourier transform because of subtle cancellations in the Fourier integration over all of the wave function, i.e. over both its superoscillating and non-superoscillating parts. We will find that to this end the amplitudes in the superoscillating part of the wave function must be significantly smaller than the amplitudes in the non-superoscillating parts.

\section{MAXIMAL SUPEROSCILLATIONS}

Our aim, therefore, is to calculate those normalized wave functions which possess the most pronounced superoscillations. The strategy will be to use a variational principle to first calculate that wave function $\psi$ of the form of Eq1 which possesses the smallest norm while 
obeying the arbitrarily chosen constraints of Eqs 2 Second, we normalize the wave function. In this way, having minimized the norm of $\psi$, we insure that after the normalization of $\psi$ the amplitudes of the superoscillations are maximal.

Explicitly, we minimize the norm $\int_{-\infty}^{\infty} \psi^{*}(x) \psi(x) d x=$ $\int_{-p_{\max }}^{p_{\max }} \tilde{\psi}^{*}(p) \tilde{\psi}(p) d p$ of the wave function $\psi$, subject to the constraints of Eq2 which can also be written as:

$$
\frac{1}{\sqrt{2 \pi \hbar}} \int_{-p_{\max }}^{p_{\max }} \tilde{\psi}(p) e^{\frac{i x_{k} p}{\hbar}} d p=a_{k}
$$

Thus, with Lagrange multipliers $\mu_{r}$, the Lagrangian of the variational problem reads

$$
\begin{aligned}
L= & \int_{-p_{\max }}^{p_{\max }} \tilde{\psi}^{*}(p) \tilde{\psi}(p) d p \\
& +\sum_{r=1}^{N} \frac{\mu_{r}}{\sqrt{2 \pi \hbar}} \int_{-p_{\max }}^{p_{\max }} \tilde{\psi}(p) e^{\frac{i x_{r} p}{\hbar}} d p+\text { c.c. }
\end{aligned}
$$

from which we obtain:

$$
\tilde{\psi}(p)+\sum_{r=1}^{N} \frac{\mu_{r}^{*}}{\sqrt{2 \pi \hbar}} e^{\frac{-i x_{r} p}{\hbar}}=0
$$

Using Eq[3] we have therefore:

$$
\frac{-1}{2 \pi \hbar} \sum_{r=1}^{N} \mu_{r}^{*} \int_{-p_{\max }}^{p_{\max }} e^{\frac{i p}{\hbar}\left(x_{k}-x_{r}\right)} d p=a_{k}
$$

We define the symmetric matrix $\left\{S_{k, r}\right\}_{k, r=1}^{N}$ through:

$$
\begin{aligned}
S_{k, r} & =\frac{1}{2 \pi \hbar} \int_{-p_{\max }}^{p_{\max }} e^{\frac{i p}{\hbar}\left(x_{k}-x_{r}\right)} d p \\
& =\frac{\sin \left(\left(x_{k}-x_{r}\right) p_{\max } / \hbar\right)}{\pi\left(x_{k}-x_{r}\right)}
\end{aligned}
$$

Note that $S$ is invertible since it is positive definite:

$$
\sum_{r, k=1}^{N} v_{r}^{*} S_{r, k} v_{k}=\frac{1}{2 \pi \hbar} \int_{-p_{\max }}^{p_{\max }}\left|\sum_{l=1}^{N} v_{l} e^{i p x_{l}}\right|^{2} d p
$$

Eq 5 now takes the form

$$
-\sum_{r=1}^{N} S_{k, r} \mu_{r}^{*}=a_{k}
$$

and we can solve for the coefficients $\mu_{r}^{*}$ :

$$
\mu_{r}^{*}=-\sum_{m=1}^{N} S_{r, m}^{-1} a_{m}
$$

Using Eq4 we finally obtain the desired wave function in the momentum space representation:

$$
\tilde{\psi}(p)=\frac{1}{\sqrt{2 \pi \hbar}} \sum_{r=1}^{N} \sum_{m=1}^{N} S_{r, m}^{-1} a_{m} e^{\frac{-i x_{r} p}{\hbar}}
$$

In the position representation, the superoscillating wave function reads:

$$
\begin{aligned}
\psi(x) & =\frac{1}{2 \pi \hbar} \int_{-p_{\max }}^{p_{\max }} \sum_{r=1}^{N} \sum_{m=1}^{N} S_{r, m}^{-1} a_{m} e^{\frac{i\left(x-x_{r}\right) p}{\hbar}} d p \\
& =\sum_{r, m=1}^{N} S_{r, m}^{-1} a_{m} \frac{\sin \left(\left(x-x_{r}\right) p_{\max } / \hbar\right)}{\pi\left(x-x_{r}\right)}
\end{aligned}
$$

We see that $\psi$ is a delicate linear combination of shifted copies of the function $\sin \left(2 \pi x / \lambda_{\min }\right) / x$ each of which is of only slow variation. Among all wave functions which obey Eq1 and which take the prescribed amplitudes $a_{k}$ at the $N$ prescribed positions $x_{k}$, the wave function given in Eq11 possesses the smallest norm. Thus, the normalized wave function $\psi^{(n)}=\frac{1}{\|\psi\|} \psi$ superoscillates with the largest achievable amplitudes:

$$
\psi^{(n)}\left(x_{k}\right)=\frac{a_{k}}{\|\psi\|}, \quad \text { for all } k=1, \ldots, N
$$

\section{SELF-ACCELERATION THROUGH SINGLE SLIT}

The properties of superoscillating wave functions are unusual:

Consider a low-momentum particle as it passes through a narrow slit in a screen. The particle's position in the direction parallel to the screen thereby becomes determined to within the width $L$ of the slit. By the uncertainty principle, this implies that the particle's momentum $\Delta p$ parallel to the screen becomes uncertain, such as to obey $\Delta p \geq \frac{\hbar}{L}$. Thus, as is well-known, a particle may acquire momentum when passing through a narrow slit.

We can now see, however, that the uncertainty principle is not the only reason why particles can acquire momentum when passing through a slit. Namely, consider an incident low-momentum particle whose wave function in the direction parallel to the screen possess spatial superoscillations just where the wave function hits the slit in the screen.

In this case, the wave function which emerges from the slit is spatially oscillating with the very short wavelength of the superoscillations where the slit is and is zero elsewhere. The emerging wave function's rapid oscillations now do show as high frequencies in its Fourier transform. This is because the emerging wave no longer possesses the high-amplitude non-superoscillating parts which cancelled the high frequencies' occurrence in the incident wave's Fourier transform. Thus, the emerging quantum particle acquires a correspondingly high momentum expectation value.

Crucially, since the superoscillating stretch of the particle's wave function can be chosen arbitrarily wide, also the width of the slit can be chosen arbitrarily wide. This shows that this new mechanism by which particles are 
accelerated when passing through a slit occurs independently from and on top of the uncertainty relation.

\section{SCALING BEHAVIOR}

Superoscillations come at a price, however, which is that in normalized wave functions any superoscillations are generally of very small amplitude, implying that particles are not very likely to be found in regions where their wave function is superoscillating.

To see this, note that due to Eq 12 the superoscillations' amplitude is suppressed by $\|\psi\|$, for which Eq4 yields:

$$
\begin{aligned}
\|\psi\|^{2} & =\frac{1}{2 \pi \hbar} \int_{-p_{\max }}^{p_{\max }}\left|\sum_{r=1}^{N} \mu_{r}^{*} e^{\frac{-i x_{r} p}{\hbar}}\right|^{2} d p \\
& =\sum_{k, r=1}^{N} \frac{\mu_{r}^{*} \mu_{k}}{2 \pi \hbar} \int_{-p_{\max }}^{p_{\max }} e^{\frac{i\left(x_{k}-x_{r}\right) p}{\hbar}} d p \\
& =\vec{\mu}^{\dagger} S \vec{\mu}
\end{aligned}
$$

We used vector notation: $\vec{\mu}=\left\{\mu_{r}\right\}_{r=1}^{N}$. Using Eq[9] we obtain:

$$
\|\psi\|^{2}=\vec{a}^{\dagger} S^{-1} \vec{a}
$$

Clearly, the largest suppression occurs when $\vec{a}$ is an eigenvector of the symmetric matrix $S^{-1}$ with the largest eigenvalue, i.e. when $\vec{a}$ is an eigenvector of the symmetric matrix $S$ with the smallest eigenvalue, say $s_{\min }$. The normalized wave function $\psi^{(n)}$ then superoscillates with the amplitudes:

$$
\psi^{(n)}\left(x_{k}\right)=s_{\min }^{1 / 2} \frac{a_{k}}{\|\vec{a}\|}
$$

Explicitly, let us prescribe the superoscillating amplitudes at equidistant points with spacing $\Delta x$, namely $x_{k}=k \Delta x$ for $k=0, \ldots,(N-1)$. In this case, we obtain a matrix $S$ whose eigenvalues were studied in [11]: The definition of the prolate matrix $\rho(N, W)$ in Eq.21 of [1] matches ours through $S_{r, k}=\rho_{r, k}(N, W(\Delta x)) / \Delta x$ with $W(\Delta x)=\Delta x p_{\max } / h$.

Eq.64 of 11] then straightforwardly yields the scaling of $s_{\text {min }}$, and thus of $\psi^{(n)}\left(x_{k}\right)$, for fixed $N$ and decreasing spacing $\Delta x$ :

$$
\psi^{(n)}\left(x_{k}\right) \propto s_{\min }^{1 / 2} \propto(\Delta x)^{N-1}
$$

Thus, if a fixed number $N / 2$ of equidistant superoscillations is compressed into a smaller region, i.e. if the superoscillations' wavelength $2 \Delta x$ is reduced, then the superoscillation amplitude decreases polynomially with the wavelength of the superoscillations.

In the case where the superoscillation wavelength, i.e. the spacing $\Delta x$, is held fixed and the number $N$ of superoscillations is increased, we can use Eqs.13,58 from
[1] to readily find the scaling behavior of the smallest eigenvalue $s_{\min }$ of $S$ for large $N$, to obtain:

$$
\psi^{(n)}\left(x_{k}\right) \propto s_{\min }^{1 / 2} \propto N^{1 / 4} e^{-\gamma N / 2}
$$

Here, $\gamma$ is positive and depends on $\Delta x$ but not on $N$. Thus, we proved that the amplitude of superoscillations decreases exponentially with the number of superoscillations, i.e. with the length of the superoscillating stretch, as had in fact been conjectured by Berry, in [2].

While these scaling results imply exceedingly small superoscillation amplitudes, we note that if the superoscillating stretch of a wave function does pass through a slit, then the emerging superoscillations' amplitudes get boosted to normal levels through the renormalization of the wave function.

\section{OPEN QUESTIONS}

The phenomenon of superoscillations raises a number of fundamental questions, which we here only begin to address:

1) In the single slit setup of above, the superoscillating particles which do pass through the slit will gain arbitrary predetermined amounts of momentum and energy. Due to momentum and energy conservation, this gain must be balanced by a corresponding loss in the momentum and energy of the screen. Thus, those particles are accelerated by strong interactions with the slit walls, which are in effect cooling the screen. Indeed, wave functions can act as "cooling rays" where they superoscillate. The energy picked up by those particles is available to do work because the particle's motion after the screen is directed, since their momentum is largely predetermined. Consistency with the second law of thermodynamics therefore requires either a) that the cooling effects of superoscillating parts of the wave functions are necessarily offset by the presence of a heating non-superoscillating part or b) that it is necessarily entropy-expensive to produce superoscillating wave functions.

2) Further, the rate at which incident particles pass through the slit and thereby take energy from the screen does not depend on the screen's temperature. How, therefore is it ensured that the screen always has sufficient energy and momentum available for those particles which happen to pass through the slit? The answer should be, although we do not prove this here, that if the position of a cold screen is known sufficiently accurately to ensure that it is the superoscillating part of incident wave functions which passes through the slit, then by the uncertainty relation the screen possesses sufficient momentum uncertainty to be able to provide the required momentum to the superoscillating particles which pass through the slit. Conversely, if the screen is known to possesses very little momentum, then by the uncertainty 
relation the position of the screen and its slit cannot be known with sufficient precision to ensure for the emerging particles that it was the superoscillating part of their wave function which passed through the slit.

3) Generally, one may expect that in a single-particle experiment, if a property of the particle can be predicted, the particle ought to have possessed that property before the measurement. However, we now know for example that if a low-momentum particle is found in a region where its wave function is rapidly superoscillating then it will be found to possess a very large and predictable momentum. Nevertheless, the particle clearly did not possess this large momentum beforehand and only acquires it in the measurement process.

4) Continuing this argument, one might expect that a superoscillating stretch of a wave function could act as a false source of gravity. Namely, a delocalized particle might locally gravitate and be curving space according to the scale of energy-momentum density that it would possess if the particle was found in that local region, rather locally gravitating according to its actual energy-momentum if there is no measurement. Such false sources of gravity should be suppressed effectively by the fact that the amplitudes in superoscillating stretches of wave functions are very low, as we saw.

5) Interestingly, our finding that the superoscillations' amplitude decreases exponentially with the length of the superoscillating stretch becomes plausible after translation into the language of information theory. Namely, instead of wave functions with a finite momentum cutoff, consider signals with a finite bandwidth. We see that by using superoscillating signals it is in principle possible to encode arbitrary amounts of information into a short interval of a low-bandwidth signal which then passes through a low-bandwidth channel of some fixed finite noise level. However, as Shannon showed in [8], signals which pass through a channel of bandwidth $\omega_{\max }$ can deliver information at most at a rate $\omega_{\max } \log _{2}(1+S / N)$, where $S / N$ is the signal-to-noise ratio. Thus, the price for superoscillatory information compression in signals is an exponential increase in the required signal power, which is consistent with our finding of an exponential increase of the functions' norm. This example suggests that if there is an actual short-wavelength cutoff for physical fields in nature, say at the Planck scale, it may usefully be thought of as a finite upper bound on information density.

6) The question arises how superoscillating wave functions might be produced experimentally. For example, can we design a potential $V(x)$ whose ground state has a superoscillating stretch? One possibility might be to start with a harmonic oscillator potential centred about $x=0$. Its ground state's momentum range is effectively cut off at high momenta since it behaves as $\propto e^{-k p^{2}}$. Let us add to the potential several relatively sharp spikes in close proximity, close to $x=0$. The ground state's wave function should then become quickly varying in the region where the spikes are, while also suppressed in amplitude. Whether this would effectively constitute a superoscillatory ground state remains to be studied.

Acknowledgement: A.K. and P.J.S.G.F. were in part supported by the Natural Sciences and Engineering Research Council of Canada (NSERC) and by the Fundação para a Ciência e Tecnologia (FCT) of Portugal respectively.

[1] Y. Aharonov, J. Anandan, S. Popescu, L. Vaidman, Phys. Rev. Lett. 642965 (1990)

[2] M. Berry, in Proc. Intl. Conf. on Fund. Aspects of Quantum Theory, Columbia, SC, USA, 10-12 Dec. 1992 Eds. J.S. Anandan, J. L. Safko, World Scientific, Singapore (1995)

[3] M. V. Berry, J. Phys. A27 L391 (1994)

[4] H. Rosu, Nuovo Cim. 112B 131 (1997), gr-qc/9606070

[5] B. Reznik, Phys. Rev. D55 2152 (1997)

[6] Y. Aharonov, B. Reznik, A. Stern, Phys. Rev. Lett. 81 2190, (1998)

[7] A. Kempf, gr-qc/9907084 J. Math. Phys. 412360 (2000)

[8] C. E. Shannon, Proceedings of the Institute of Radio Engineers, 37, (1) 10 (1949)

[9] J.J. Benedetto, P.J.S.G. Ferreira, Modern Sampling Theory, Springer Verlag, Heidelberg (2001)

[10] L. Levi, IEEE Trans. Inform. Theory, 11, 372 (1965)

[11] D. Slepian, Bell Sys. Tech. Journ. 57, 1371 (1978) 